\title{
Gait Analysis for Human Identification by using BPNN with LDA and MDA Classifiers
}

\author{
Ira Gaba \\ Computer Science Department \\ Indo Global College of Engineering, PTU \\ Chandigarh, India
}

\author{
Satinder Pal Ahuja, PhD \\ Computer Science Department \\ Indo Global Group of Collages, PTU \\ Chandigarh, India
}

\begin{abstract}
In this paper BPNN performance for the gait analysis with using the different modulation techniques i.e LDA and MDA. Gait Analysis is the new technique in the biometric identification, the gait have more advantages over the field of biometric systems like face recognition, finger printing etc. Gait Analysis is a method by which individual can be recognized by the manner of walk. Less unobtrusive gait recognition system over the other biometric traits is the main advantage. i.e. it offers the identification of an individual at a particular distance, without any physical interference or contact with an individual and can be easily apply on the low resolution image frames as well. In this paper, firstly the video of an individual in captured, secondly background subtraction is applied on that so as to remove the unwanted information, thirdly feature extraction is carried out to extract the various parameters by using the Hanavan's model, and finally the recognition is performed by using BPNN+LDA and BPNN+MDA techniques, are used for the training and the testing purposes, and the matching can also be performed on the basis of CBIR. All the processes are performed on the gait database and the input video.
\end{abstract}

\section{Keywords}

Back-propagation neural network(BPNN),CBIR, Feature extraction, Gait recognition system, linear discriminant Analysis (LDA) and multilinear discrimant analysis(MDA).

\section{INTRODUCTION}

The Gait Recognition system is the one in which the biometric identification can be performed on the manner by which an individual walks. Gait is defined by a manner an individual's walk. And the systematic study of the human walking is known as Gait Analysis. The human walk starts approximately at the age of one years, and becomes fully established by three and half in normal case. The basic normal gait pattern leads to reduction in efficiency and increase in energy consumption. So, analysis of gait has became a topic of interest among a wide range of professionals, particularly clinical gait analysis, sports biomechanics, neurology and rehabilitation. The human walk involves the entire body head, trunk, arms, hand, hip, legs and feet working in synchronization with each other, leading to the forward propulsion of the whole human system. Divergent to conventional understanding, it is not merely the skeleton that walks, but a whole-body web of tissues, stretching and contracting to harness the potential energy available. Gait-based recognition for human identification is relatively a new research arena in biometrics. Basic motive is to discriminate individual's walk from others. So far, gait is a probably the only perceivable biometric feature from a far distant recognition. Every individual have a particular way of walking, which is useful for the biometric point of view. Human ambulation consists of synchronized movements with integrated efforts of hundreds of muscles and joints[1]. Biometric is a renown field of technology that uses automated methods for identifying and verifying a person on the basis of the physiological and behavioral traits[2].

\section{GAIT RECOGNITION SYSTEM}

The gait recognition system[3] is a system which is used to identify the gait of an individual, by comparing the gait of an individual with the sequence of data stored in the database. The proposed gait recognition system is shown in the figure1. In this system we firstly, convert the captured video into image frames, secondly the background subtraction is performed on the image frames so as to eliminate the non-usable information. After that feature extraction is performed to extract various parameters such as distance between the right hand and the left leg, distance between the head and the feet(the height of the individual), distance between both the hands, distance between the right leg and the left hand, length of the one leg and the length of the one hand can be implement on the basis of hanavan's model. CBIR is also performed for the matching purposes. And lastly the particular individual is recognized on the basis of BPNN with LDA and MDA.

Step 1: Capture the video for the identification of the individual.

Step 2: Background Subtraction is performed on the input video to remove the unwanted information.

Step 3: Detection and Tracking is performed after that. In this the location of the subject is obtained.

Step 4: Various parameters like distance between the right hand and left leg, distance between the head and the feet(the height of the individual), distance between both the hands, distance between the right leg and the left hand, length of the one leg and the length of the one hand have been calculated for the feature extraction, and the model which is used here is Hanavan's model.

Step 5: After calculating the various parameters the gait recognition is carried out.

Step 6: The matching is performed on the basis of Content Based Image Retrieval.

Step 7: After that recognition is performed on the basis of Neural Network Techniques.

The below figure 1. shows the basic layout of the proposed gait recognition system. All the steps which have to be carried out for the identification of an individual is shown above. 


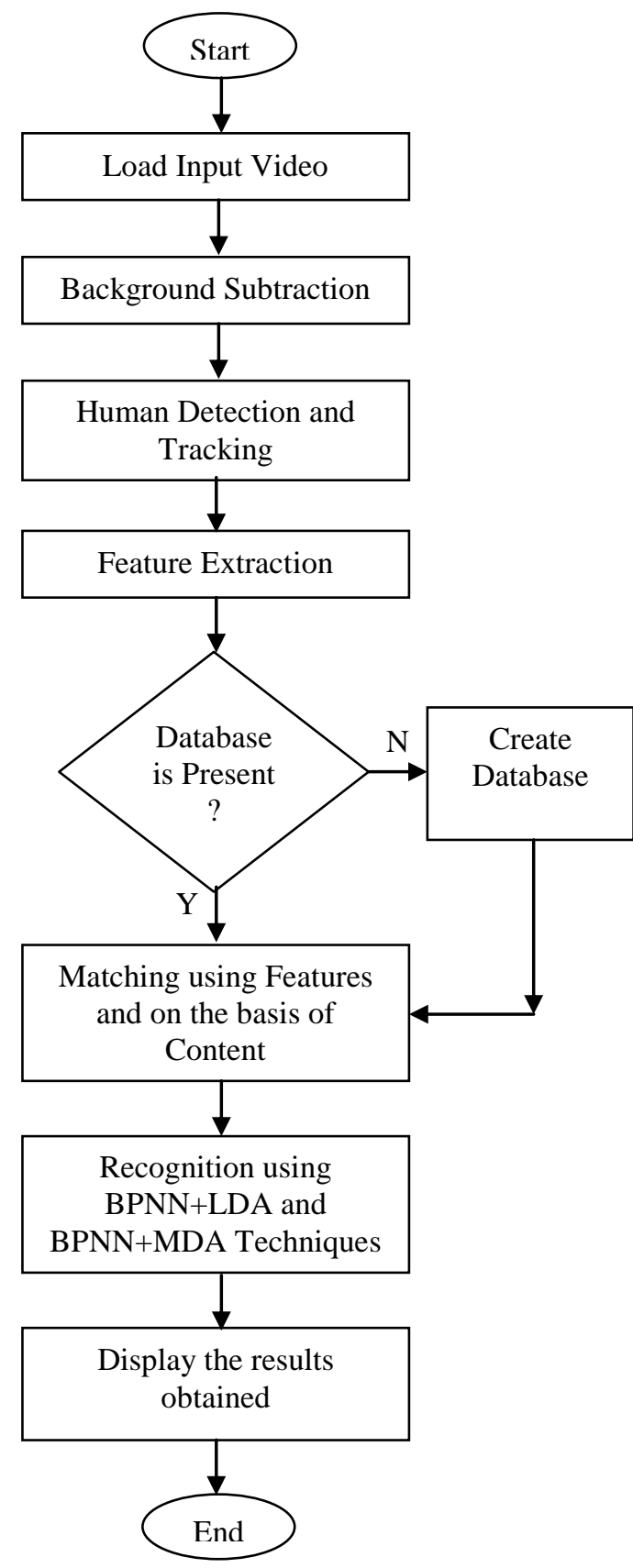

Figure 1. Flowchart of the Proposed System

\subsection{Capture the Input Video}

Initially an input video is captured for the gait identification, further then that input video is converted into the frames which are commonly known as video sequences and after that these frames are further used for the recognition process.

The first step to be carried out is to capture the videos having the person's walking. The following are the assumptions during the process of video capturing:

- The device for capturing video have to be stationary at the time of video capture.

- The only moving object in the frame of view is the subject, while the video is being recorded.

- The line of view of the device is at right angles to the direction of walking of the subject.
The devices which are used for the video capturing should have a sufficient clarity of resolution. It is preferable that within a single database all videos were recorded from the same distance, this is requirement for uniformity of feature parameters.

\subsection{Background Subtraction}

The second step after converting video into frames, is of background subtraction. The basic use of the background subtraction is to remove or omit all the unwanted information i.e. only to retain the useful data. Generally the primary and the vital task for many computer-based applications is to recognize the moving objects from a video sequence. Background subtraction is a common approach, which identifies the moving objects from a portion of a video frame, that significantly differs from a background model. By Background subtraction[4], the required information can be retained. The background subtraction is perform, in which the moving objects is recognize from the section of the video frame that differs from the background model. It generates binary images which contains white and black moving pixels know as binary silhouettes.

Background Subtraction is carried out on the video frames, to reduce the presence of noise. The basis common method for motion detection is Background Subtraction . The following equation shows the calculation for the background subtraction is simple and easy to implement.

$D_{k}(x, y)=\left\{\begin{array}{cr}1 \text { if }\left|F_{k}(x, y)-B_{k-1}(x, y)\right|>T \\ 0 & \text { otherwise }\end{array}\right\}$

where $D_{k}(x, y)$ is the resultant difference, $F_{k}(x, y)$ is the current frame of the system and $B_{k-1}(x, y)$ is the background initialized frame and $\mathrm{T}$ is the threshold which hold back the shadow depending on the value assigned in the system.

There are many other different ways for calculation the foreground image by background subtraction[5]. Median filter can be used after background subtraction to remove the remaining noises, some noises may remain present due to bad background subtraction.

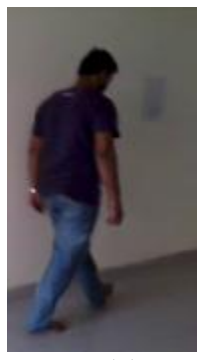

(a)

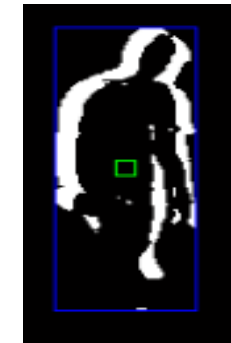

(b)
Figure 2. (a)the original input image (b)

Foreground image calculated by using the matlab image processing toolbox, by subtracting the current image with background

The outcome of the background subtraction is a image that maps the difference between background image and the original input image. And to extract the silhouette, the difference image have to be converted into a binary image by implementing suitable threshold value into the pixels different image, which further results into a binary image that reflects a entire different image, and the required image can be filtered without any loss of information.

\subsection{Detection and Tracking.}

Detection and Tracking are computer vision systems based tasks used for locating and following person in a video sequence. Human detection and tracking are considered to be the first two 
processes for the video surveillance and further feed into higherlevel reasoning modules such as recognition and static/dynamic scene analysis.

The object tracking is used in the foreground image to determine the position and the other relevant information of the moving objects in the sequences and to map out the object frame by frame. The main motive is to obtain the binary image of the silhouette[6],[7] that is nearly matching the actual silhouette of the walking person. The main aim of tracking is to subtract the two subsequent frames, in which the part of images which doesn't change can get subtracted to give the zero intensity i.e. the background image gets subtracted and after subtraction, it gives the black pixels. On the other hand, only the moving object don't get reduces to zero because the intensity of the moving object of the two subsequent frames are different.

To generate the silhouette following steps have to be carried out.

1. Firstly, background subtraction is to be done, on the input video, frame by frame. therefore the resultant image is to be binaries into the foreground(white) and the background(black) pixels

2. A boundary box is placed around the moving image that contains the moving person, as that part of the image as shown in the figure 2(b). therefore, in this the size of the box is chosen to accommodate all the information in the database.

3. The left and the right boundaries of the body are traced. Subsequently the height of the silhouette is obtained in terms of numbers and saved in the database.

By this, the height of the silhouette can be calculated and this will further help in calculating the various parameters. The parameters calculated can further used in for the recognition purposes.

\subsection{Feature Extraction}

Feature Extraction is the next step which is very important in the gait analysis process. The feature must be robust to operating conditions and good discriminability should be yield across the individual. Basically feature extraction is the process in which salient features can be extracted, that will effectively captures the gait characteristics. Transformation of the input data into the particular features(parameters) is known as feature extraction.

Feature extraction can be done by two ways[8]: Model-based approach and Model-free approach.

In the model based approach, Initially binary silhouette is obtained by the background subtraction. And the some of the features can be extracted from it such as the static and the dynamic information by tracking body the various components such as the arms, thighs, legs and limbs. In this approach, various parameters can be calculated on the requirement of the system.

Where as in the Model-free approach the focus is either on the shape of the silhouette or the motion of the individual. The Model-based approach models the whole body or any part of the body. The Model-free approach do not form the structure of the human motion.

In feature extraction by using the model-based approach, various parameters can be calculated such as distance between the left leg and right hand, distance between head and feet(the eight of the individual), distance between both hands, distance between right leg and left hand, length of the one leg and the length of the one hand can be implement on the basis of hanavan's model.

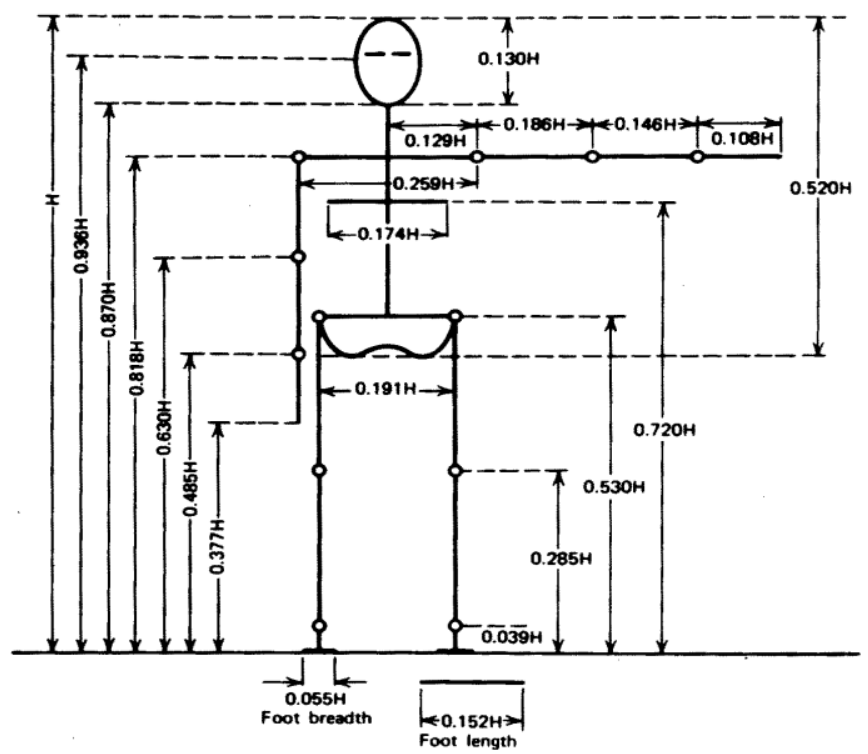

Figure 3. Body segment length expressed as a function of body height

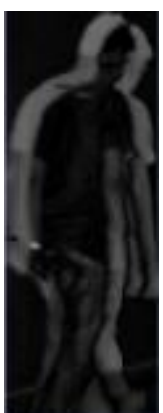

(a)

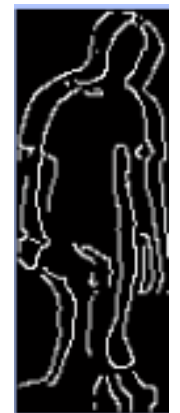

(b)

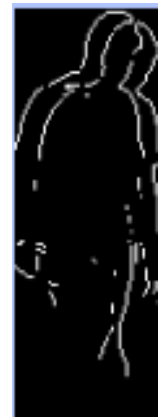

(c)
Figure 4. (a)The foreground image. (b)The image calculated by using matlab image toolbox(canny method). (c)The image calculated by using matlab image toolbox(prewitt method)

By the help of the Hanavan's model, various parameters can be calculated, which further help in the recognition part.

Various images can be calculated by using the matlab image toolbox as shown in the above figure 4. Different method of the matlab can be used for calculating various forms of the images.

\subsection{Recognition}

Recognition is the most important and the critical step for the gait-based human identification. In this, the input test video sequences are compared with the trained sequence in the database.

CBIR[9][10][11] is used for the matching purposes. In CBIR the images are retrieved on the basis of the automatic derived features. The images can be described objectively, in the Content based image retrieval by a set of low-level features such as texture and shape etc. In this we use the SURF method to improve the retrieval efficiency. SURF(Speed up robust feature) is one of the most popular interest point detector. Most of the computer vision based applications uses the SURF method only.

Back-Propagation[12] Algorithm is a multilayer network consist of the output layer, hidden layer and the output layer. It follows the supervised learning method and use the expert edge or extrapolate the delta rule. It further works on acquiring a dataset of the desired output from various inputs, in provision of making the training set. It is most valuable for the networks that do not 
have feedback, or simply, which do not have a connections. The back propagation requires the activation function(known as the nodes) used by the artificial neurons.

In this firstly the propagation is carried out, in this forward propagation is results to produce the output activations and the back-propagation from output activations through the neural network is done to produce the deltas of all the output and the hidden neurons. Secondly weight update for each, by multiplying its delta output and input activation to obtain the gradient and by bring the weight in the opposite direction of the gradient by subtracting a ratio of it from the weight.

For learning rule, consider the simple case of a neuron $(\mathrm{k})$ constituting the only computational node in the output layer of a neural network. Neuron $(\mathrm{k})$ is subset of a signal vector $\mathrm{x}(\mathrm{n})$ produced by one or more layers of hidden neurons, which themselves are again a subset of an input vector applied to the source nodes (i.e. input layer) of the neural network. The argument ' $n$ ' denotes discrete time function, the time frame of an iterative process involved in adjusting the synaptic weights of neuron (k). The output signal of neuron $(\mathrm{k})$ is denoted by $y_{k}(n)$. This output signal, which represents the output of the neural network, is compared with desired response or target output, denoted by $d_{k}(n)$. An error signal $e_{k}(n)$. we thus have

$e_{k}(n)=d_{k}(n)-y_{k}(n)$

The error signal $e_{k}(n)$ actuates a control mechanism, the purpose of which is to apply sequence of corrective adjustments to the synaptic weights of neuron $(\mathrm{k})$. The corrective adjustments are designed to make the output signal $y_{k}(n)$ more closer to the desired response $d_{k}(n)$ in a step-by-step sequent manner. This objective of sequential correction is to achieve the minimal cost function $\mathrm{E}(\mathrm{n})$, defined in terms of the error signal $e_{k}(n)$ as

$\mathrm{E}(\mathrm{n})=\frac{1}{2} e_{k}^{2}(n)$

The minimization of the cost function $\mathrm{E}(\mathrm{n})$ leads to a learning rule commonly referred to as a delta rule.

Linear Discriminant Analysis(LDA)[14][15][16] is a well known module used for the feature extraction in recognition and for dimension reduction.

Given a data matrix $\mathrm{A} \in \mathrm{I} R^{N x n}$, classical LDA aims to find a transformation $\mathrm{G} \in \mathrm{I} R^{N x l}$ that maps each column $a_{i}$ of $\mathrm{A}$, for $1 \leq \mathrm{i} \leq \mathrm{n}$, in the $\mathrm{N}$-dimensional space to a vector $b_{i}$ in the 1dimensional space. That is $\mathrm{G}: a_{i} \in \mathrm{I} R^{N} \rightarrow b_{i}=G^{t} a_{i} \in \mathrm{I} R^{l}(1<$ $\mathrm{N})$. Equivalently, classical LDA aims to find a vector space $G$ spanned by $\left\{g_{i}\right\}_{i=1}^{l}$, where $\mathrm{G}=\left[g_{1}, \ldots, g_{l}\right]$, such that each $a_{i}$ is projected onto $G$ by $\left(g_{1}^{T} . a_{i}, \ldots, g_{l}^{T} a_{i}\right)^{T} \in \mathrm{I} R^{l}$.

Assume that the original data in $\mathrm{A}$ is partitioned into $\mathrm{k}$ classes as $\mathrm{A}=\left\{\Pi_{1}, \ldots, \Pi_{k}\right\}$, where $\Pi_{i}$ contains $n_{i}$ data points from the ith class, and $\sum_{i=1}^{k} n_{i}=n$. LDA aims to find the optimal transformation $G$ such that the class structure of the original high-dimensional space is preserved in the low-dimensional space.

In general, if each class is grouped in sophisticatedldefined pattern, but well separated from the each other, then the quality of the cluster is rated as high. In discriminant analysis, two main scatter matrices are known as, within-class $\left(S_{w}\right)$ and between$\operatorname{class}\left(s_{b}\right)$ matrices, are defined to measure the quality, as below
$S_{w}=\sum_{i=1}^{k} \sum_{x \in \Pi_{i}}\left(x-m_{i}\right)\left(x-m_{i}\right)^{T}$

and

$S_{b}=\sum_{i=1}^{k} n_{i}\left(m_{i}-m\right)\left(m_{i}-m\right)^{T}$

where $m_{i}=\frac{1}{n_{i}} \sum_{x \in \Pi_{i}} x$ is the mean of the ith class, and $\mathrm{m}=\frac{1}{n} \sum_{i=1}^{k} \sum_{x \in \Pi_{i}} x$ is the global mean.

The various notations which are used are shown in the following table 1 .

Table 1. Notations

\begin{tabular}{|c|l|}
\hline Notation & Description \\
\hline $\mathrm{N}$ & number of images in the dataset \\
\hline $\mathrm{K}$ & number of classes in the dataset \\
\hline$A_{i}$ & ith image in the matrix representation \\
\hline$a_{i}$ & ith image in the vectorized representation \\
\hline$\Pi_{j}$ & jth class in the dataset \\
\hline $\mathrm{L}$ & transformation matrix(left) \\
\hline $\mathrm{R}$ & transformation matrix(right) \\
\hline $\mathrm{I}$ & number of iterations \\
\hline
\end{tabular}

Multi-Linear Discriminant Analysis[17][18](MDA) is generally a dimensionality reduction framework. It can prevent from the dimensionality dilemma by using k-mode optimization approach and higher order tensors. The problem of small size sample is also resolved by this approach. In general, the extension from vector to tensor for data representation and feature extraction. And it can be applied many other algorithms to improve the algorithmic learnability and effectiveness.

Given the sample set $\mathrm{X} \in R^{m_{1} \times m_{2} \times \ldots \times m_{n} \times N}$, their class labels $c_{i} \in\left\{1,2, \ldots, N_{c}\right\}$ and the final lower dimensions $m_{1}^{\prime} \times m_{2}^{\prime} \times$ $\ldots \times m_{n}^{\prime}$.

1. Initialize $U_{1}^{0}=I_{m_{1}}, U_{2}^{0}=I_{m_{2}}, \ldots U_{n}^{0}=I_{m_{n}}$;

2. For $\mathrm{t}=1,2, \ldots T_{\max }$ do

a) For $\mathrm{k}=1,2, \ldots, \mathrm{n}$ do

$Y_{i}=X_{i} \times_{1} U_{1}^{t} \ldots U_{k-1} U_{k-1}^{t} \times_{k+1} U_{k+1}^{t-1} \ldots \times_{n} U_{n}^{t-1}$

$Y_{i}^{k} \Leftarrow_{k} Y_{i}$

$S_{B}=\sum_{j=1}^{\Pi_{0 \neq k}^{m_{0}}} S_{B}^{j}, S_{B}^{j}=\sum_{c=1}^{N_{c}} n_{c}\left(\bar{Y}_{c}^{k, j}-\bar{Y}^{k, j}\right)\left(\bar{Y}_{c}^{k, j}-\bar{Y}^{k, j}\right)^{T}(6)$

$S_{W}=\sum_{j=1}^{\Pi_{0 \neq k}^{m_{0}}} S_{W}^{j}, S_{W}^{j}=\sum_{i=1}^{N}\left(Y_{i}^{k, j}-\bar{Y}_{c_{i}}^{k, j}\right)\left(Y_{i}^{k, j}-\bar{Y}_{c_{i}}^{k, j}\right)^{T}$

$$
S_{B} U_{k}^{t}=S_{w} W_{k}^{t} A_{k}, \quad U_{k}^{t} \in R^{m_{k} \times m_{k}^{\prime}}
$$

(b) if $\mathrm{t}>2$ and $\left\|U_{k}^{t}-U_{k}^{t-1}\right\|<m_{k}^{\prime} m_{k} \in, \mathrm{k}=1, \ldots, \mathrm{n} \quad$ break;

3. Output the projections $U_{k}=U_{k}^{t} \in R^{m_{k} \times m_{k}^{\prime}}, \mathrm{k}=1, \ldots, \mathrm{n}$.

The graphical representation of the above methods are shown in the end in the figure 5. and figure 6.

\section{CONCLUSION}

The computer vision techniques make assured that vision based techniques can be acquired. Paper described here provides simplified methodology for the human identification. 
Background subtraction method used to trace and segment the silhouette of the individual under surveillance. Many other Gait analysis techniques discussed here in subject as well, but the combination of the BPNN, LDA and MDA for the recognition generates the better results. Experimental Results is widely observed in the high accuracy rate which indicates the robust outermost contour feature and feature extraction model used is also effective.

The best accuracy $98.8 \%$ achieved and the comparisons with the state-of-the-art gait recognition methods proves that the proposed method is a very promising gait recognition method in gait recognition community.

\section{FUTURE SCOPE}

1. Acquiring gait characteristics from single camera based setup have some limitations. However multiple camera based setup having significant advantages.

2. Performance rate has to be improved by occlusion, clothing style conditions and different walking considerations.

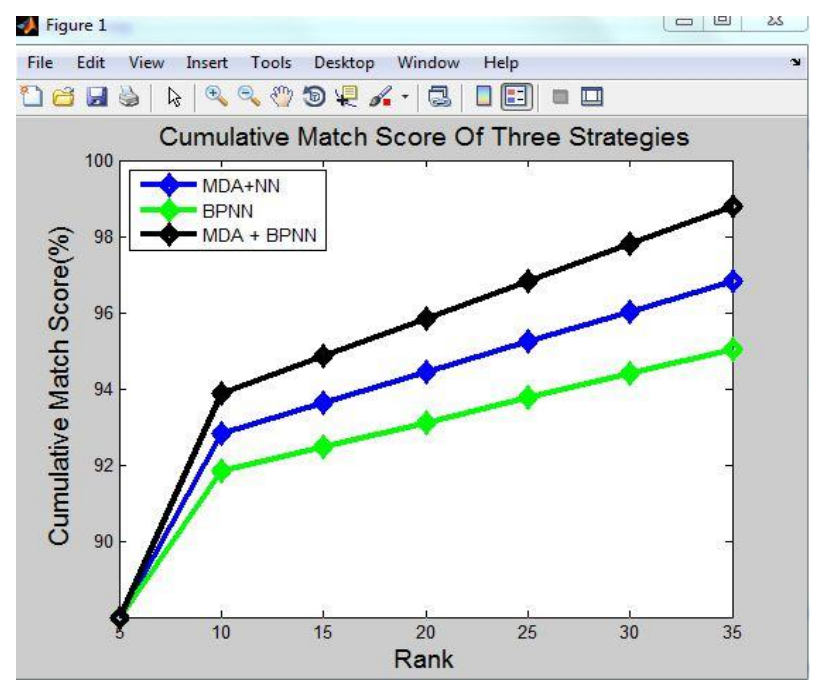

Figure 5 The graph between the MDA+NN and MDA+BPNN

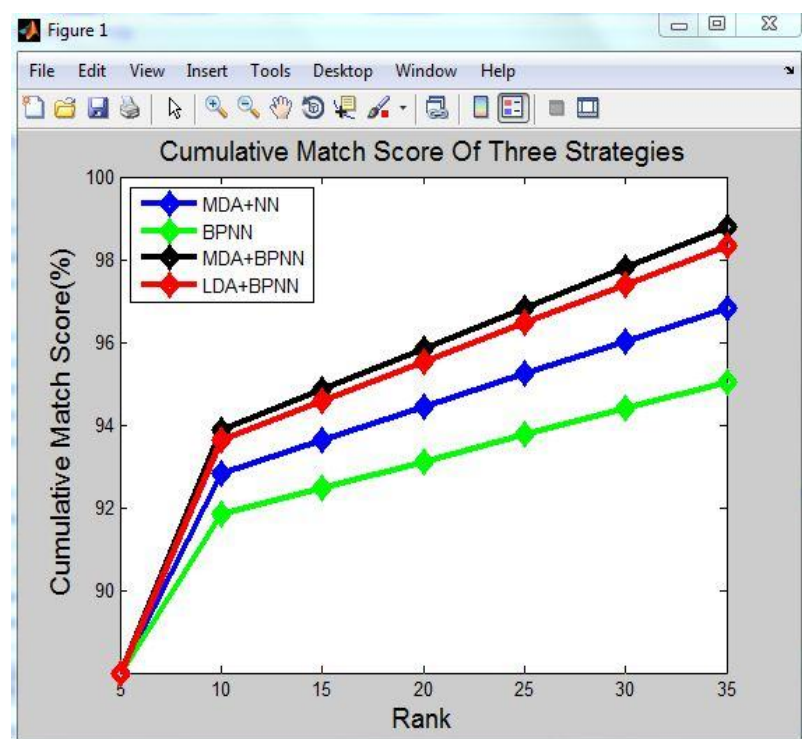

Figure 6. The final graphical representation of all the techniques used

\section{REFERENCES}

[1] C. BenAbdelkader, R. Culler and L. Davis, "Stride and Cadence as a Biomertic in Automatic Person Identification and Verification" in Proceeding International Conference Automatic Face and Gesture Recognition, pp.372-377, 2002.

[2] Qinghan,"Technology review- Biometrics Technology, Application, Challenge and Computational Intelligence Solution",IEEE Computational Intelligence Magazine, Vol 2,pp5-25,2007.

[3] Boulgouris, N.V Plataniotis, K.N Hatzinakos, “ An Angular Transform of Gait Sequences for Gait Assisted Recognition", In: Proc.IEEE int. Conf. Image Processing Singapore, pp. 857-860, 2004.

[4] M. Pushparani, D. Sasikala, "A Survery of Gait Recognition approach using PCA and ICA", Global Journal of Computer Science and Technology Network, Web \& Security, Vol. 12, Issue 10, Version 1.0, May 2012.

[5] Lili Li, Yilong Yin, Wei Qin, Ying Li, “Gait Recognition Based on Outer Contour",in International Journal Of Computational Intelligence Systems, Vol.4,No.5, pp. 10901099, March2012.

[6] A Hayder, J.Dargham, A. Chekima and G.M. Ervin, "Person Identification Using Gait", in International Journal of Computer and Electrical Engineering, Vol. 3, No. 4, pp. 477-482, August 2011

[7] Liang Wang, Tieniu Tan, Huazhong Ning and Weiming $\mathrm{Hu}$, "Silhouette Analysis - Based Gait Recognition for Human Identification" IEEE transactions on Pattern Analysis and Machine Intelligence, Vol. 25, No. 12, December 2003

[8] Jin Wang, Mary She, Saeid Nahavandi, Abbas Kouzani, "A Review of Vision-Based Gait-Recognition methods for Human Identification, in DICTA: Proceedings of the Digital Image Computing: Techniques and Application, IEEE, Piscataway,pp. 320-327, 2010.

[9] Stevan Rudinac, Goran Zajic, Marija Uscumlic and Maja Rudinac, "Comparison of CBIR Systems with Different Number of Feature Vector Components", in IEEE Second International Workshop on Semantic Media Adaptation and Personalization, pp. 199-204, 2007.

[10] Jianlin Zhang and Wensheng Zou, "Content-based Image Retrieval Using Color and Edge Directions Features ", in IEEE pp. 456-462, 2010.

[11] K. Velmurugan, Dr. S. Santhosh Baboo,“ Content- Based Image Retrieval using SURF and Color Moments ",in Global Journal of Computer Science and Technology, Vol. 11, Issue 10, 2011.

[12] Ernest Istook, Tony Martinez, ,“ Improved Backpropagation Learning in Neural Networks with Windowed Momentum", in International Journal of Neural Systems, Vol. 12, no. 3\&4, pp. 303-318.

[13] A. A. Minai, and R.D. Williams, ,“ Acceleration of Backpropagation Through Learning Rate and Momentum Adaption ", in International Joint Conference on Neural Networks, IEEE, pp 676-679, 1990.

[14] Shuiwang Ji, and Jieping Ye, “" Generalized Linear Discriminant Analysis: A Unified Framework and Efficient Model Selection", in IEEE transactions on Neural Networks, Vol. 19, No. 10, October 2008

[15] Dong Xu, Yi Huang, Zinan Zeng and Xinxing Xu, ,“ Human Gait Recognition Using Patch Distribution Feature 
and Locality-Constrained Group Sparse Representation ", in IEEE Transactions on Image Processing, Vol. 21, No. 1, January 2012.

[16] Deng Cai, Xiaofei He, and Jiawei Han, ," SRDA An Efficient Algorithm for Large-Scale Discriminant Analysis", in IEEE transactions on knowledge and Data Engineering, Vol. 20, No. 1, January 2008
[17] Shuicheng Yan, Dong Xu, Qiang Yang Lei Zhang Xiaoou Tang and Hong-Jiang Zhang, "“ Multilinear Discriminant Analysis for face recognition",in IEEE Transactions on Image Processing, Vol.16, No. 1 January 2007.

[18] Tao.Li, Shenghuo.Zhu, and Mitsunori. Ogihara, ,“ Using discriminant for multi-class classification: an experimental investigation ",In Knowledge and Information Systems, Vol. 10, pp453-472, 2006. 